\title{
Orbital Angular Momentum (OAM) Mode-Reconfigurable Discrete Dielectric Lens Operating at $300 \mathrm{GHz}$
}

\author{
Geng-Bo Wu, Student Member, IEEE, Ka Fai Chan, Member, IEEE, Shi-Wei Qu, Senior Member, \\ IEEE, Kin Fai Tong, Senior Member, IEEE and Chi Hou Chan, Fellow, IEEE
}

\begin{abstract}
A novel 3-dimensional (3-D) printed discrete dielectric lens (DDL) antenna with orbital angular momentum (OAM) mode reconfigurability operating at $300 \mathrm{GHz}$ is proposed in this paper. The antenna consists of a stationary lower DDL and an upper in-plane rotatable DDL, fed by a stationary pyramidal horn. The DDL pair can transform the quasi-Gaussian beam from the feed source into vortex waves, and its OAM mode number, $l$, can be dynamically reconfigured among $0, \pm 1$, and \pm 2 by a simple mechanical in-plane rotation of the upper DDL panel with specific angles relative to the stationary lower one. For the DDL design, the height of each dielectric post pixel element is tuned to realize the required transmission phase. The OAM mode-reconfigurability of the proposed terahertz (THz) antenna was validated by both simulations and measurements. The simulated OAM mode purity bandwidth of the design DDL antenna are $40 \%, 31.3 \%$ and $25.8 \%$ for the OAM mode $l=0,+1$ and +2 , respectively. In addition, the $\mathrm{THz}$ line-of-sight link experiments show that acceptable mode isolations among different OAM channels can be achieved by the THz DDL antenna, validating its application for OAM mode diversity.
\end{abstract}

Index Terms-Orbital angular momentum (OAM), reconfigurable, terahertz (THz), 3-D printing, OAM mode purity bandwidth.

\section{INTRODUCTION}

$\mathrm{O}$ RBITAL angular momentum (OAM) beam, possessing a phase vortex around its beam axis, has attracted tremendous interest firstly in optical regime and later at radio frequencies since its discovery by Allen et al. in 1992 [1]. One of the appealing characteristics of an OAM beam is its inherent orthogonality among eigenstates with different topological

This work was supported in part by the Hong Kong Research Grants Council under Grant T42-103/16-N and CityU 11250216 and the Natural Science Foundation of China (NSFC) under Grant 61622104.

G. B. Wu, and C. H. Chan are with the State Key Laboratory of Terahertz and Millimeter Waves, Department of Electrical Engineering, City University of Hong Kong (CityU), Hong Kong, China (corresponding to: eechic@,cityu.edu.hk ).

K. F. Chan is with the State Key Laboratory of Terahertz and Millimeter Waves, City University of Hong Kong (CityU), Hong Kong, China.

S. W. Qu is with the School of Electronic Science and Engineering, University of Electronic Science and Technology of China (UESTC), Chengdu 611731, China.

K. F. Tong is with the Department of Electronic and Electrical Engineering, University College London, London, WCIE 7JE, U. K. charges, which means OAM-based mode-division multiplexing (MDM) technique opens another diversity scheme for larger channel capacity in additional to the conventional frequency, polarization, and space multiplexing. Although it has been proven as a subset of multiple-input-multiple-output (MIMO) techniques [2], OAM beam can also be used to increase the channel capacity and spectral efficient for short range wireless communications, such as terahertz $(\mathrm{THz})$ ultra-high speed line-of-sight links.

Various OAM beam generators have been proposed in past decades. Spiral phase plates (SPP) is the most commonly used structure to transform the Gaussian beam into OAM beam both in microwave [3] and optical [4]-[5] domains. SPPs are dielectric refractive components whose thickness increases in proportion to the azimuth angle to provide helical phase distribution. SPP can also be realized using a flat phase plate with different equivalent permittivities by drilling inhomogeneous holes [6]. Planar structures based on split-ring frequency selective surfaces [7] and metasurfaces [8] are developed to emulate the functionality of SPP. Reflective type transform devices such as twisted parabolic reflectors [9]-[10] and reflectarrays [11] are also reported. Other OAM beam launcher includes uniform circular arrays [12]-[14], dielectric resonator antennas [15]-[16], time-switched arrays [17]-[18] and traveling-wave antennas [19], etc.

Although significant research efforts have been made to generate OAM beams, most reported OAM generator can only generate fixed OAM mode. In the applications of wireless communications using direct OAM index modulation [20] and vortex imaging [21]-[23], different OAM waves are needed and mode-reconfigurable devices capable of generating switchable OAM modes are highly demanded to improve the system performance. Circular array with reconfigurable feeding network using $\mathrm{p}-\mathrm{i}-\mathrm{n}$ diodes is the most common method to generate multiple switchable OAM modes at microwave frequencies [24]-[27]. However, the complex and lossy feeding networks as well as the shortage of high frequency diodes prohibit these structures to be used at THz frequencies. In [28], a mechanically reconfigurable circular array using spiral antenna element is designed at microwave frequencies. In this configuration, actuators are added to every antenna element and a feeding network is also required. Graphene-based reflectarray antenna [29]-[30] is a good candidate for tunable OAM beam 


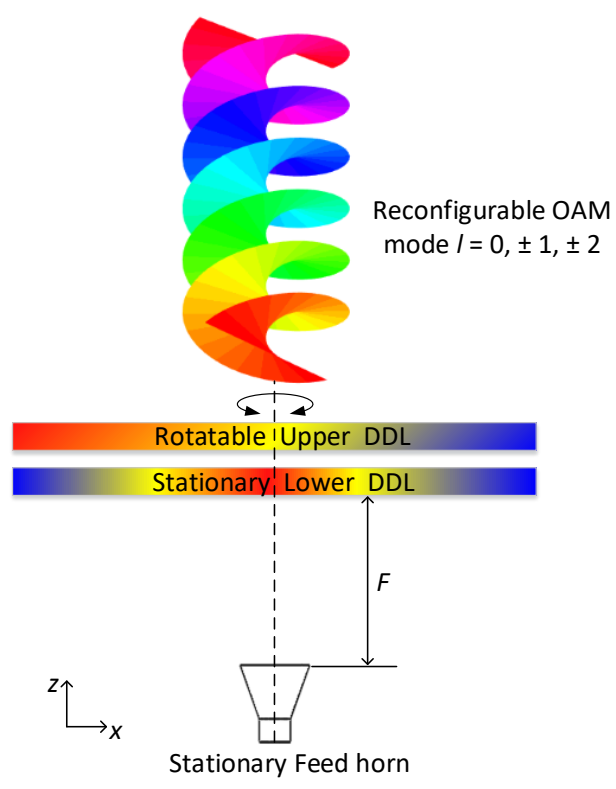

Fig. 1. Schematic of the OAM mode-reconfigurable DDL antenna.

generation at $\mathrm{THz}$ frequencies but no experimental results have been demonstrated so far.

In this paper, we propose a novel OAM mode-reconfigurable discrete dielectric lens (DDL) antenna operating at $300 \mathrm{GHz}$. DDL [31]-[33] is an appealing antenna structure for $\mathrm{THz}$ applications since no complicated feeding network is needed due to its space feeding nature. DDL can individually control the transmission phase of each pixel element and thereby realize arbitrary aperture phase distribution. As a result, DDL can emulate the conventional bulky SPP to transform the Gaussian beam into vortex beam but with lower profile and reduced dielectric loss. Moreover, the polarization of the DDL can be controlled by combining with artificial dielectric grating [34]. When compared with its reflectarray counterpart [35]-[37], DDL can avoid the feed horn blockage and exhibits better tolerance to fabrication error. Different from other phase-shifting structures [6]-[8], DDL is compatible with 3-dimensional (3-D) printing technology and hence can be realized with rapid prototyping and low cost. The proposed OAM mode-reconfigurable antenna is composed of two thin DDLs fed by a stationary feed horn. Similar to the Risley prisms [38]-[39], mechanical in-plane rotation is adopted in this antenna structure. The two DDLs are designed such that they can transform the quasi-Gaussian beam from the feed source into vortex waves, whose OAM mode number depends on the in-plane rotation angle of the upper lens relative to the lower one. Both simulated and measured results are provided to demonstrate the mode reconfigurability of the proposed $\mathrm{THz}$ DDL antenna.

\section{Operating Principle}

The schematic of the proposed mode-reconfigurable DDL antenna is illustrated in Fig. 1. A stationary feed source in this design is desirable since movements of the RF modules and electronic interconnections in $\mathrm{THz}$ band can be avoided. It consists of two thin parallel DDLs fed by a stationary feed horn.
To generate vortex radio waves with OAM mode number $l$, the required total compensation phase distribution of the two DDLs, $\phi(\rho, \varphi)$, should be:

$$
\phi(\rho, \varphi)=k_{0} \sqrt{F^{2}+\rho^{2}}-l \varphi+C
$$

The first term on the right-hand side in (1) is to compensate for the spatial phase delay from the phase center of the feed source to the position of the pixel element, the second term $-l \varphi$ is to generate the desired vortex wavefront above the aperture, while $C$ is a phase constant added to all the elements on the aperture. $k_{0}$ is the free-space wavenumber at the operating frequency of $300 \mathrm{GHz}, F$ is the focal length, i.e., spatial distance between the phase center of the feed source to the geometric center of the DDL. $(\rho, \varphi)$ are the polar coordinates of the pixel element of the lens.

Assume that the initial compensation phase distributions (i.e., without in-plane rotation) of the lower and upper DDLs are $\phi_{L}(\rho, \varphi)$ and $\phi_{U}(\rho, \varphi)$ respectively. To realize reconfigurable OAM mode, the lower and upper DDLs are in-plane rotated clockwise by the angles of $\varphi_{L}$ and $\varphi_{U_{2}}$ respectively. Therefore, the value of the mode number $l$ in (1) should be the function of $\varphi_{L}$ and $\varphi_{U}$. The sum of the compensation phase profiles of the lower and upper DDLs should satisfy the required phase profile in (1) to generate the twisted wave, i.e.,

$$
\begin{aligned}
& \phi_{L}\left(\rho, \varphi+\varphi_{L}\right)+\phi_{U}\left(\rho, \varphi+\varphi_{U}\right) \\
& =k_{0} \sqrt{F^{2}+\rho^{2}}-l\left(\varphi_{L}, \varphi_{U}\right) \varphi+C
\end{aligned}
$$

Since the first term on the right-hand side in (2) is independent of $\varphi$, the compensation phase profiles of the lower and upper DDLs are constructed as:

$$
\begin{aligned}
\phi_{L}(\rho, \varphi) & =\frac{1}{2} k_{0} \sqrt{F^{2}+\rho^{2}}+f(\varphi) \\
& =\frac{1}{2} k_{0} \sqrt{F^{2}+\rho^{2}}+a_{0}^{L}+a_{1}^{L} \varphi+a_{2}^{L} \varphi^{2}+a_{3}^{L} \varphi^{3}+\cdots \\
\phi_{U}(\rho, \varphi) & =\frac{1}{2} k_{0} \sqrt{F^{2}+\rho^{2}}+g(\varphi) \\
& =\frac{1}{2} k_{0} \sqrt{F^{2}+\rho^{2}}+a_{0}^{U}+a_{1}^{U} \varphi+a_{2}^{U} \varphi^{2}+a_{3}^{U} \varphi^{3}+\cdots
\end{aligned}
$$

The $\varphi$-dependent terms $f(\varphi)$ and $g(\varphi)$ for the lower and upper DDL compensation phase profiles are further written as polynomial expansion in (3) and (4). $a_{i}^{L}$ and $a_{i}^{U}$ are the undetermined coefficients of the $i$ th-order polynomials for the lower and upper DDLs, respectively. To determinate these coefficients, we assume that the DDL antenna generates OAM number $l=0$ in its initial state without rotation of the two DDLs, i.e., $\left(\varphi_{L}, \varphi_{U}\right)=\left(0^{\circ}, 0^{\circ}\right)$. Substituting (3) and (4) into (2), we obtain

$$
\begin{gathered}
k_{0} \sqrt{F^{2}+\rho^{2}}+\left(a_{0}^{L}+a_{0}^{U}\right)+\left(a_{1}^{L}+a_{1}^{U}\right) \varphi+\left(a_{2}^{L}+a_{2}^{U}\right) \varphi^{2} \\
+\left(a_{3}^{L}+a_{3}^{U}\right) \varphi^{3}+\cdots=k_{0} \sqrt{F^{2}+\rho^{2}}+C
\end{gathered}
$$

Comparing term by term in (5), one obtains

$$
a_{i}^{L}=-a_{i}^{U}, \text { for } i \geqslant 1
$$

When the lower and upper DDLs are rotated to generate high-order OAM modes, the total compensation phase profile 
of the two DDLs can be obtained by combining (3), (4) and (6):

$\phi_{\text {tot }}=\phi_{L}\left(\rho, \varphi+\varphi_{L}\right)+\phi_{U}\left(\rho, \varphi+\varphi_{U}\right)$

$=k_{0} \sqrt{F^{2}+\rho^{2}}+\sum_{i=0}^{\infty} a_{i}^{L}\left(\varphi+\varphi_{L}\right)^{i}+\sum_{i=0}^{\infty} a_{i}^{U}\left(\varphi+\varphi_{U}\right)^{i}$

$=k_{0} \sqrt{F^{2}+\rho^{2}}+a_{0}^{L}+a_{0}^{U}+\sum_{i=1}^{\infty} a_{i}^{L}\left[\left(\varphi+\varphi_{L}\right)^{i}-\left(\varphi+\varphi_{U}\right)^{i}\right]$

$=k_{0} \sqrt{F^{2}+\rho^{2}}+a_{0}^{L}+a_{0}^{U}+a_{1}^{L}\left(\varphi_{L}-\varphi_{U}\right)+a_{2}^{L}\left[2 \varphi\left(\varphi_{L}-\varphi_{U}\right)+\varphi_{L}^{2}-\varphi_{U}^{2}\right]$

$+a_{3}^{L}\left[3 \varphi^{2}\left(\varphi_{L}-\varphi_{U}\right)+3 \varphi\left(\varphi_{L}^{2}-\varphi_{U}^{2}\right)+\varphi_{L}^{3}-\varphi_{U}^{3}\right]+\cdots$

Substituting (7) into (2) and comparing term by term, one can find that the coefficients should satisfy the following relations:

$$
\begin{gathered}
a_{0}^{L}+a_{0}^{U}+a_{1}^{L}\left(\varphi_{L}-\varphi_{U}\right)+a_{2}^{L}\left(\varphi_{L}^{2}-\varphi_{U}^{2}\right)=C \\
2 a_{2}^{L}\left(\varphi_{L}-\varphi_{U}\right)=-l \\
a_{i}^{L}=0, \text { for } i \geqslant 3
\end{gathered}
$$

$C$ is a reference phase constant, which is independent of $(\rho, \varphi)$. It does not change the phase gradient among elements and has no influence on the OAM generation. As a result, one can freely select different values of the reference phase $C$ for different OAM cases. To simplify the compensation phase profile of the DDLs, $C$ is chosen as

$$
C=a_{2}^{L}\left(\varphi_{L}^{2}-\varphi_{U}^{2}\right)
$$

Substituting (11) into (8), one obtains $a_{1}^{L}=0$, and $a_{0}^{L}=-a_{0}^{U}$. Therefore, the compensation phase profiles of the lower and upper DDLs in (3) and (4) can be simplified as:

$$
\begin{aligned}
& \phi_{L}(\rho, \varphi)=\frac{1}{2} k_{0} \sqrt{F^{2}+\rho^{2}}+a_{2}^{L} \varphi^{2}+a_{0}^{L} \\
& \phi_{U}(\rho, \varphi)=\frac{1}{2} k_{0} \sqrt{F^{2}+\rho^{2}}-a_{2}^{L} \varphi^{2}-a_{0}^{L}
\end{aligned}
$$

It can be seen that $a_{0}^{L}$ is the reference phase constant of the DDL and it is set as 0 in practice. The total compensation phase of the two DDLs in (7) can be written as

$$
\begin{aligned}
\phi_{t o t} & =\phi_{L}\left(\rho, \varphi+\varphi_{L}\right)+\phi_{U}\left(\rho, \varphi+\varphi_{U}\right) \\
& =k_{0} \sqrt{F^{2}+\rho^{2}}+2 a_{2}^{L} \varphi\left(\varphi_{L}-\varphi_{U}\right)+a_{2}^{L}\left(\varphi_{L}{ }^{2}-{\varphi_{U}}^{2}\right) \\
& =k_{0} \sqrt{F^{2}+\rho^{2}}-l^{\prime} \varphi+C
\end{aligned}
$$

where

$$
l^{\prime}=2 a_{2}^{L}\left(\varphi_{U}-\varphi_{L}\right)=2 a_{2}^{L} \Delta \varphi
$$

From (14), it can be observed that combination of the two DDLs can generate the desired total phase distribution as in (1) to transform the spherical phase-front from the feed source into the transmitted vortex wavefront. From (15), the azimuthal mode index of the DDL antenna $l$ ' depends on the relative rotation angle between the lower and upper DDL $\Delta \varphi=\varphi_{U}-\varphi_{L}$, which can be changed to realize different OAM mode numbers. To simplify the whole antenna structure, the lower lens is kept fixed (i.e., $\varphi_{L}=0^{\circ}$ ) and only the upper lens is mechanically rotated such that only one actuator is required in practice. Note that the compensation phase profile of the upper DDL after rotation can be written as $\phi_{U}\left(\rho, \varphi+\varphi_{U}\right)$ only in the azimuth range $\varphi \in\left[0,2 \pi-\varphi_{U}\right]$ due to the phase ambiguity. As a result, the derivation (7)-(15) holds only in the

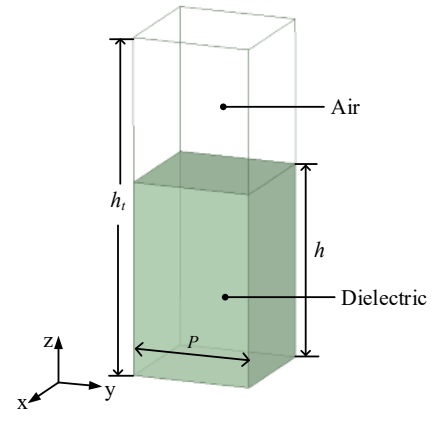

(a)

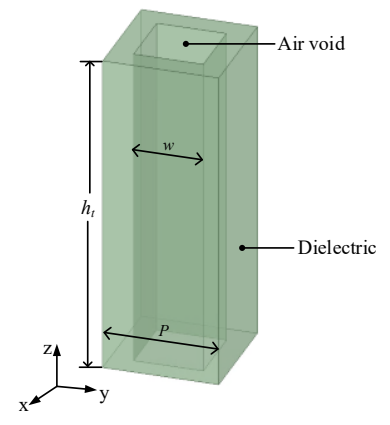

(b)
Fig. 2. Configuration of the dielectric pixel element: (a) height-variable type and (b) transverse-variable type.

azimuth range $\varphi \in\left[0,2 \pi-\varphi_{U}\right]$. For the azimuth range $\varphi \in\left[2 \pi-\varphi_{U}, 2 \pi\right]$, total transmission phase of the two DDLs will deviate from the desired one in (1), but this does not have a significant effect for small physical rotation angle cases or small OAM mode numbers.

To generate the desired integer OAM mode number, the physical rotation angle of the upper DDL is:

$$
\varphi_{U}=\frac{l^{\prime}}{2 a_{2}^{L}}, \quad l^{\prime}=0, \pm 1, \pm 2, \ldots
$$

It can be seen that the constant $a_{2}^{L}$ determines the required physical rotation angle of the upper DDL panel to switch its operating OAM mode number. A larger value of $a_{2}^{L}$ has larger available switchable OAM modes, but suffers from degraded radiation performance due to the sharp azimuthal phase variation with large difference in dimensions of element between surrounding neighbor elements, which will be shown later to be a significant factor on the radiation performance of the DDL antenna. When $\varphi_{U}=0^{\circ}$, the DDL antenna can transform the spherical phase-front into plane wavefront, corresponding to an OAM mode number $l=0$. Clockwise and anticlockwise rotations of the upper lens panel correspond to generate positive and negative topological indexes, respectively.

\section{Element Design}

Elements of the DDL can be generally arranged into two categories, i.e., height-variable type [31]-[33] and transverse-variable type [40]-[41] as shown in Figs. 2(a) and (b), respectively. In fact, their operating principle are similar, i.e., changing the volume fraction of the dielectric in a given cuboid space $\left(P \times P \times h_{t}\right)$ to change its effective dielectric constant and hence transmission phase. The lattice size of the dielectric pixel element is $P=0.5 \mathrm{~mm}$ (corresponding to $0.5 \lambda_{0}$, where $\lambda_{0}$ is the free-space wavelength at $300 \mathrm{GHz}$ ) and the total height of element $h_{t}=1.8 \mathrm{~mm}$. The height $h$ and width of the air hole $w$ for two kinds of elements can be varied from pixel to pixel to realize desired phase shifts. The material of the dielectric is high temperature resin [42] with a relative dielectric constant $\varepsilon_{r}=2.66$ and loss tangent of 0.03 at $300 \mathrm{GHz}$, which is measured using the EKSPLA THz time-domain 


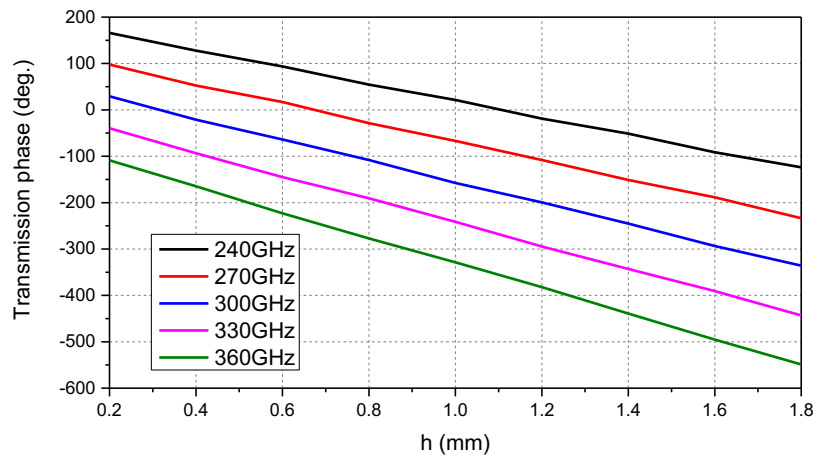

(a)

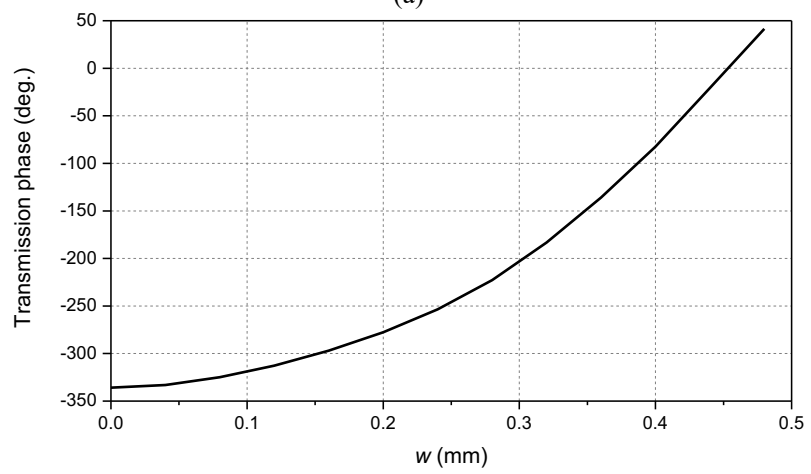

(b)

Fig. 3. Simulated transmission phase as a function of the height $h$ and width of the air hole $w$ for (a) height-variable type and (b) transverse-variable type elements.

spectroscopy and vector network analyzer (VNA) frequency-domain setups in [43]-[44]. High temperature resin is chosen as the printing material in this design for its high resistance to scratching and deformation. Other materials with a higher dielectric constant and lower loss tangent like composite acrylonitrile butadiene styrene (ABS)-based material (PREPERM ® TP20280) [45]-[46] can also be adopted for 3-D printing to reduce the thickness and dielectric loss of the lens if they can be utilized by the 3-D printer to meet the printing resolution required.

Then elements surrounding by periodic boundary conditions (PBC) are simulated in Ansys HFSS simulator to evaluate their transmission responses. The simulated transmission phase as a function of the height $h$ and width of the air hole $w$ are shown in Figs. 3(a) and (b), respectively. It can be observed that a full transmission phase range over $360^{\circ}$ can be achieved for both types of DDL elements. However, the antenna structure that can be used in $\mathrm{THz}$ band is significantly limited by the fabrication constrains. The calculated average dimension sensitivities for the height-variable and transverse-variable elements, defined as $\frac{1}{N} \sum_{i=1}^{N} \frac{\partial \phi_{i}}{\partial L_{i}}$ (where $L$ is the variable

geometrical dimension of the elements and $N$ is the total considered number of the elements), are $0.21^{\circ} / \mu \mathrm{m}$ and $0.85^{\circ}$ $\mu \mu \mathrm{m}$, respectively. It can be observed that the sensitivity of transverse-variable type element is around fourfold of its height-variable counterpart to the fabrication inaccuracy, meaning a small fabrication error could significantly change the transmission phase. Therefore, height-variable element is
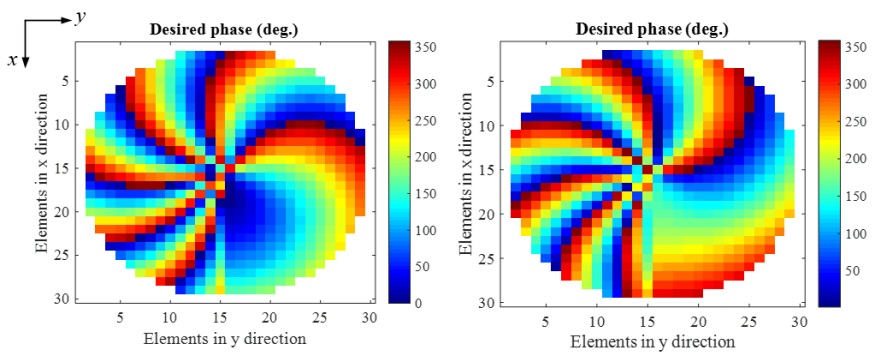

(a)

(b)

Fig. 4. Calculated desired compensation phase distributions for (a) lower lens and (b) upper lens, respectively.

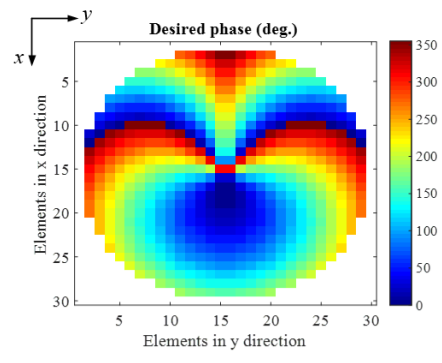

(a)

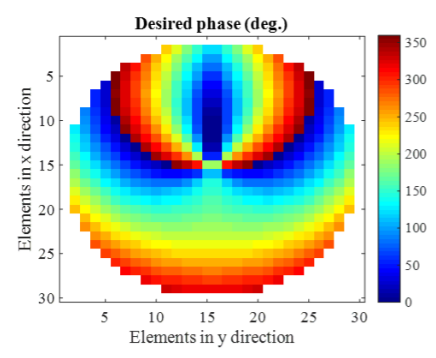

(b)
Fig. 5. Calculated modified desired compensation phase distributions for (a) lower lens and (b) upper lens, respectively.

adopted as the element for the THz DDL antenna in this work. In practice, antireflection structures, i.e., rectangular air voids are added in the top part of the dielectric post to reduce the impedance mismatch between the air and dielectric interface [31]-[33]. Fig. 3(a) also gives the simulated transmission phase of the DDL element at different frequencies from 240 to 360 GHz. It can be observed that the transmission phase curves of the dielectric post are parallel at different frequencies, indicting its broadband performance.

\section{Mode-Reconfigurable DDL Antenna Design}

In the following proof-of-concept example, a 684-element circular DDL antenna with a diameter of $15 \mathrm{~mm}$ is designed based on the mechanism introduced in Section II. A commercially available linearly polarized (LP) standard pyramid horn (Millitech SGH-03) with a gain of $23 \mathrm{dBi}$ at 300 $\mathrm{GHz}$ is adopted as the feed source. The distance between the phase center of the feed horn and the lower DDL is set as $F=24$ $\mathrm{mm}$ to achieve a proper aperture illumination. The constant $a=$ 1 is chosen in this design and the required physical rotation angle of the upper DDL to switch to the neighboring OAM mode number is $\pm 28.6^{\circ}$ according to (7). The required compensation phase distributions of the lower and upper DDLs calculated by (2)-(3) are shown in Figs. 4(a) and (b), respectively. It can be observed that windmill-shaped phase distributions are desired for the two DDLs. Meanwhile, several phase wraps along the azimuth direction, especially in the left-half region can be observed due to the quadratic relationship between the required compensation aperture phase and azimuthal angle in (2)-(3). For the height-variable dielectric element, the shadow effect between Fresnel zones is serious since significant difference in dimensions between surrounding elements occurs [47]. The approximations in the 


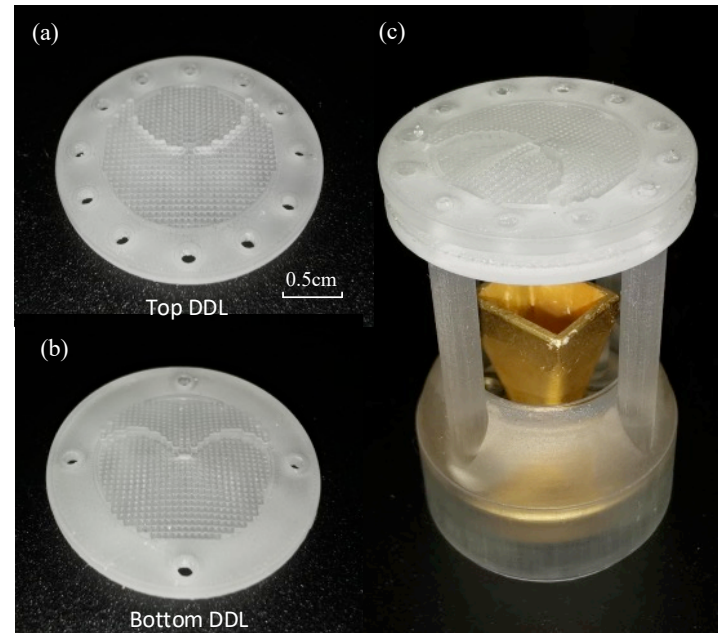

Fig. 6. Photograph of (a) the 3-D printed top DDL, (b) bottom DDL and (c) the whole OAM mode-reconfigurable DDL antenna.

pixel element simulation using infinite periodic array in Section III impose significant error in transmission coefficients. Therefore, a smooth aperture phase distribution with minimized number of zones (or phase wraps) is preferred to minimize the shadow effects. From (2)-(3), one can find that the required compensation phase distributions for both DDLs are even function of the azimuth angle $\varphi$, i.e.,

$$
\phi_{L, U}(\rho, \varphi)=\phi_{L, U}(\rho,-\varphi)
$$

Therefore, we can replace the coordinates $(\rho, \varphi)$ of the left-half region with $(\rho,-\varphi)$ to achieve a smoother aperture phase variation and the calculated revised phase distributions of the two DDLs are depicted in Fig. 5. Smoother aperture phase distributions can be obtained for both lenses and hence a better radiation performance of the whole OAM generator is expected. Then the height of each dielectric post for the two DDLs can be determined using the transmission phase curve in Fig. 3(a).

\section{Simulation and Experimental Demonstration}

To verify its mode reconfigurability, the synthesized DDL antenna is modelled and simulated in Ansys HFSS. Furthermore, the lens pair is fabricated and measured to demonstrate its OAM mode-reconfigurability. Both lenses are manufactured by the SLA 3-D printing technology using a Form2 3-D printer from Formlabs. The 3-D printer has a laser spot size of $140 \mu \mathrm{m}$ and a highest $z$-axis resolution of $25 \mu \mathrm{m}$. An in-house developed 3-D printing method demonstrated in [37] was adopted to successfully realize the $\mathrm{THz}$ dielectric lenses. The 3-D printed DDL is solid with $100 \%$ infill to reduce the thickness of the lens. Moreover, no fabrication errors occur in the printing of infill pattern for a 100\% infill. Fig. 6 shows the photograph of the 3-D printed THz DDL antenna. Good profiles of the 3-D printed lenses can be observed. Simulated results show that misalignment of the two DDLs will shift the location of the phase singularity and amplitude null of the generated vortex wave. The misalignment tolerance of the two DDLs should be smaller than $0.5 \mathrm{~mm}$ to avoid the radiation performance of the fabricated prototype deviating from that of

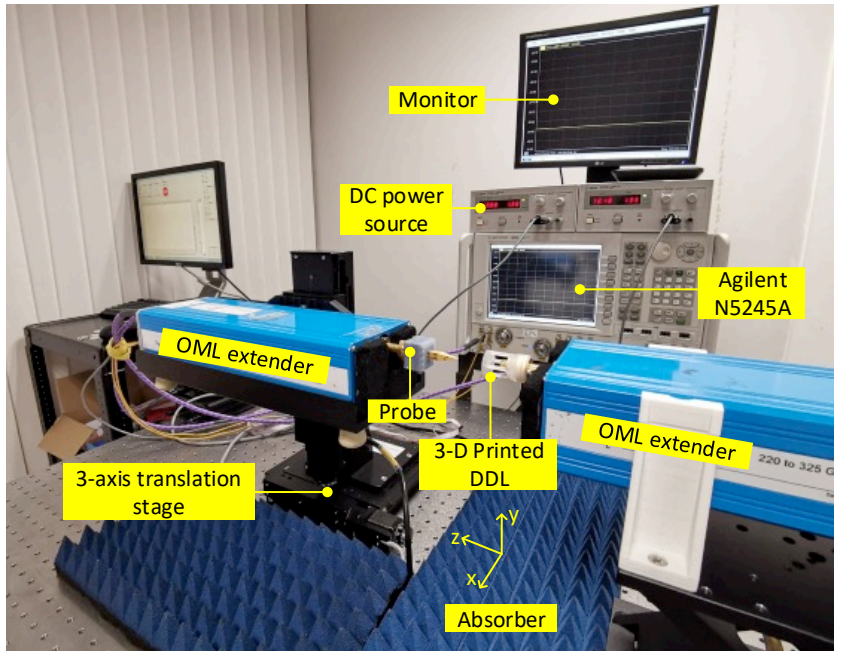

Fig. 7. Photograph of the THz near-field measurement setup.

the ideally designed one significantly. Location holes on the periphery of the two DDLs as shown in Fig. 6 are designed to realize accurate alignment of the two lenses. The space between the two lenses is $0.5 \mathrm{~mm}$. A 3-D printed fixture with four vertical posts is also fabricated to support and assemble the two lenses as shown in Fig. 7(a). In this proof-of-concept prototype, the upper DDL is simply manually rotated in the corresponding step angles, i.e., $\pm 28.6^{\circ}$ to switch the OAM mode number, while in real-world applications, rotary stage can be employed to achieve fast OAM mode reconfiguration.

Fig. 7 shows the THz near-field scanning measurement setup for testing the radiation performance of the 3-D printed DDL antenna. Two frequency extenders (OML V03VNA2-T/R) are utilized to extend the operating frequency of the VNA (Agilent $\mathrm{N} 5245 \mathrm{~A}$ ) up to $220-325 \mathrm{GHz}$. A probe is connected to the left extender installed on a 3-axis electric translational stage to record the radiation field from the antenna under test (AUT) which is connected to the right extender. A piece of radiation absorbent material (Eccosorb LS-30) is placed on the front metallic surface of the two extenders to mitigate the possible multiple reflections. The extenders and translational stage are placed on an optical table with vibration control (Newport S-2000 Stabilizer). The scanning plane is located $50 \mathrm{~mm}$ away from the prototype antenna.

Fig. 8 plots the orientation of the DDL and the corresponding simulated and measured amplitude and phase of the electric field on the scanning plane in different OAM mode numbers $l=$ $-2,-1,0,+1$ and +2 at $300 \mathrm{GHz}$. Good agreement between the simulation and measurement results can be observed. The obtained field distributions suggest that the generated beams feature the typical characteristics of the OAM modes. Specifically, for the OAM mode $l=0$, a nearly uniform phase distribution can be achieved in $-3 \mathrm{~dB}$ main beam region. The phase distributions for mode number $l= \pm 1, \pm 2$ feature a consecutive $2 \pi$ and $4 \pi$ angular phase shift along a full concentric circle, respectively. Two phase singularities for the case $l= \pm 2$ can be observed, which are caused by the non-ideal compensation phase distribution of the DDL pair. As presented 
(a) $l=-2$ :

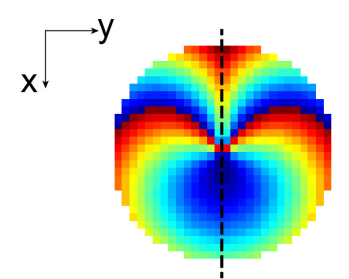

Lower lens $\varphi_{L}=0^{\circ}$

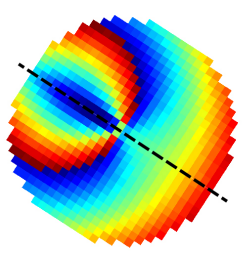

Upper lens $\varphi_{U}=-57.2^{\circ}$

(b) $l=-1$ :

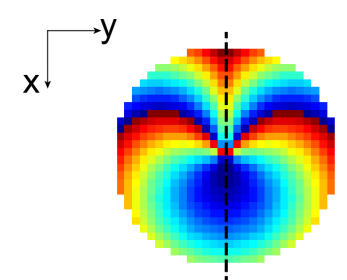

Lower lens

$\varphi_{L}=0^{\circ}$

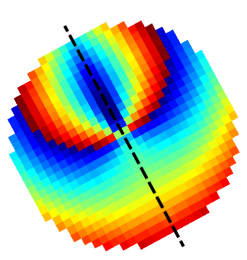

Upper lens

$\varphi_{U}=-28.6^{\circ}$

(c) $l=0$ :

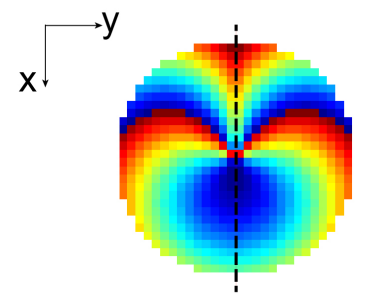

Lower lens

$\varphi_{L}=0^{\circ}$

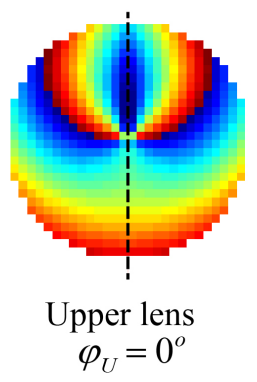

(d) $l=+1$ :

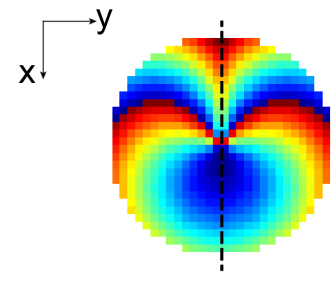

Lower lens $\varphi_{L}=0^{\circ}$

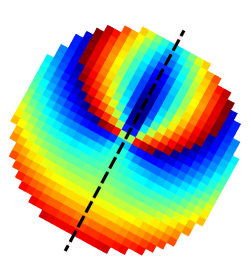

Upper lens $\varphi_{U}=28.6^{\circ}$
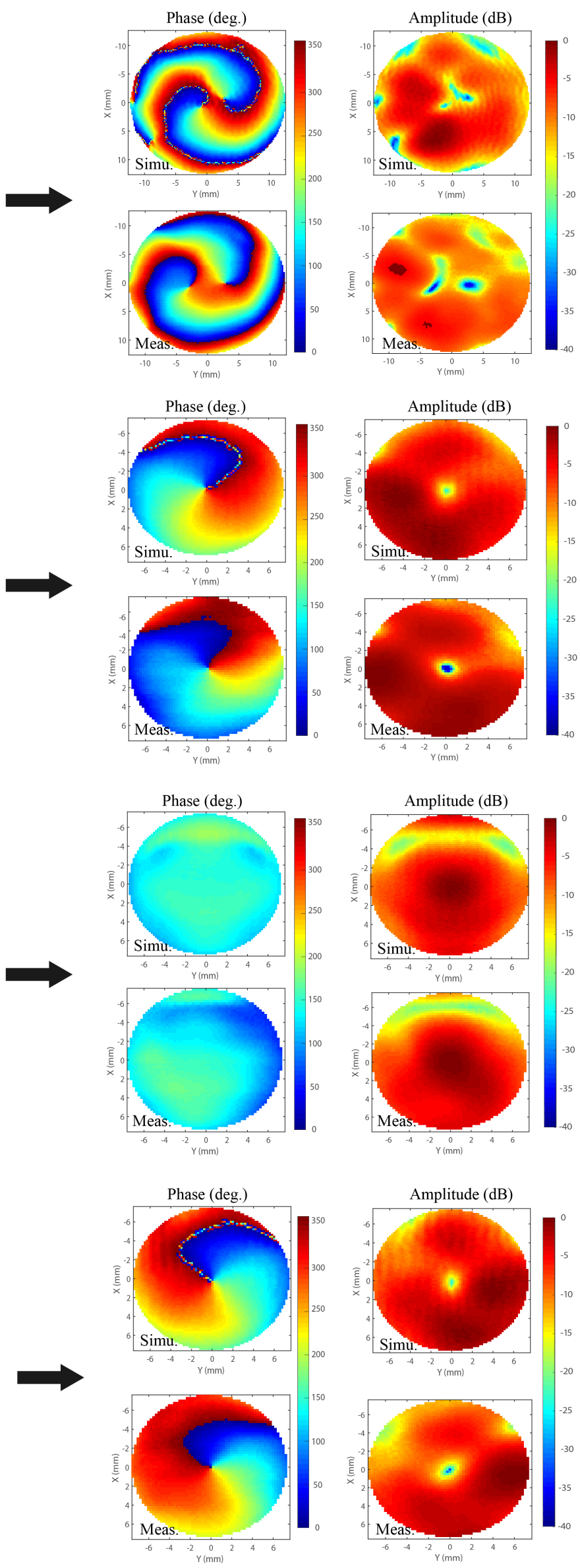
(e) $l=+2$ :

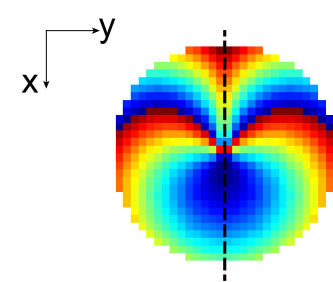

Lower lens $\varphi_{L}=0^{\circ}$

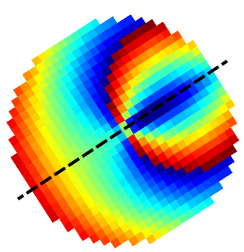

Upper lens $\varphi_{U}=57.2^{\circ}$
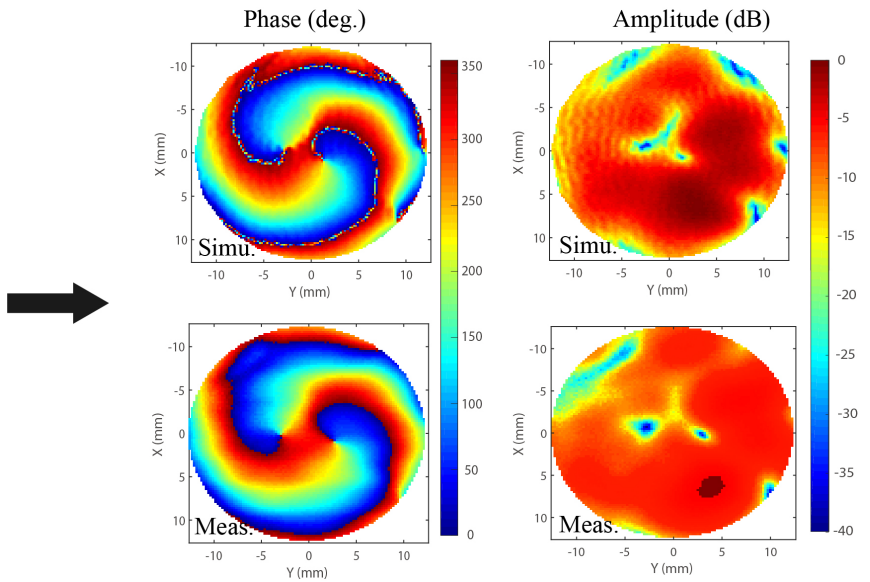

Fig. 8. Orientations of the lenses and their corresponding simulated and measured phase and amplitude distributions on the scanning plane for OAM mode number (a) $l=-2$, (b) $l=-1$, (c) $l=0$, (d) $l=+1$ and (e) $l=+2$, respectively.

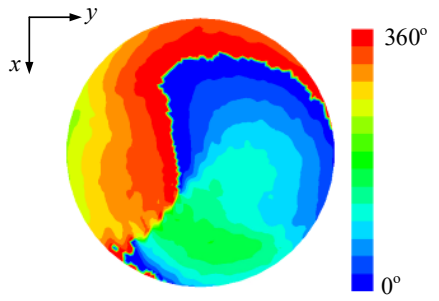

(a)

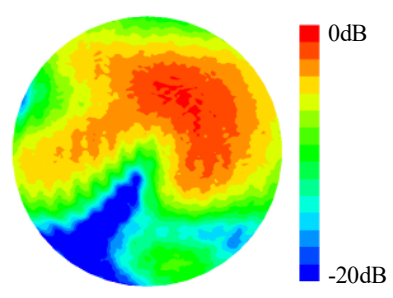

(b)

Fig. 9. Simulated (a) phase and (b) amplitude distributions of electric fields for the DDL antenna synthesized using the aperture distributions in Fig. 4 with OAM mode number $l=+1$.

in Section II, the total realized compensation phase of the two DDLs in (4) only satisfies the required compensation phase in (1) in the azimuth range $\varphi \in\left[0,2 \pi-\varphi_{U}\right]$. Specifically, the azimuth ranges of accurate compensation phase distribution for cases $l=0,1$ and 2 are $\left[0,360^{\circ}\right],\left[0,331.4^{\circ}\right]$ and $\left[0^{\circ}, 302.8^{\circ}\right]$, respectively. As a result, larger aperture phase errors occur for the case $l= \pm 2$ and form two phase singularities. The amplitude distributions in Fig. 8 show a doughnut shape with a center null overlapping with the phase singularity for modes $l= \pm 1, \pm 2$. The asymmetric amplitude distribution is caused by the asymmetric structure of the DDL antenna structure.

The simulated main beam directions of the DDL antenna are at $0^{\circ}, 3.4^{\circ}$ and $5.8^{\circ}$ with peak gains of $26.9,23.5$ and $20.3 \mathrm{dBi}$ for OAM mode number $l=0, \pm 1$, and \pm 2 , respectively. The simulated radiation efficiency of the antenna is about $54.7 \%$ at $300 \mathrm{GHz}$, which can be improved by choosing other dielectric materials with lower dielectric loss. The designed DDL antenna exhibits higher gain when compared to other reported OAM mode-reconfigurable antennas demonstrated to date [19]-[23], which is preferred to obtain the stable vortex wave as it propagates, especially in the $\mathrm{THz}$ band in this study. These numerical and experimental results reveal that the 3-D printed DDL antenna successfully generates the reconfigurable $\mathrm{THz}$ OAM vortex waves. The upper lens could be further rotated to generate higher OAM modes, but simulated results show that the radiated field deteriorates significantly when the mode order is higher than 3 . This is because higher OAM modes

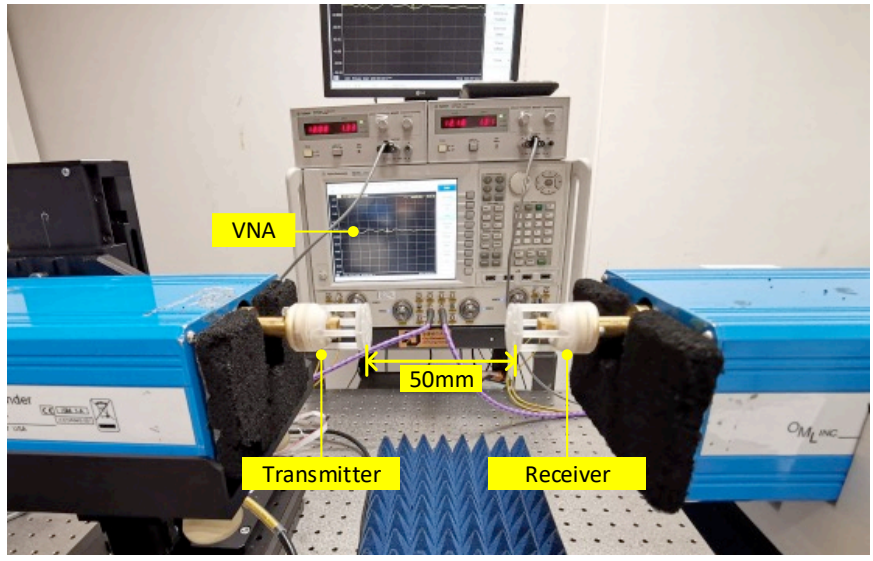

Fig. 10. Experimental setup of the THz OAM near-field line-of-sight link.

correspond to larger physical rotation angles of the upper DDL with larger phase errors in the azimuth range $\varphi \in\left[2 \pi-\varphi_{U}, 2 \pi\right]$ as explained in Section II.

For comparison purpose, a DDL antenna synthesized by the original phase distributions in Fig. 4 is also designed and simulated in Ansys HFSS. Fig. 9 plots the simulated amplitude and phase distributions of the electric field at a distance of 50 $\mathrm{mm}$ when the upper lens is rotated to realize OAM mode $l=+1$ $\left(\varphi_{U}=28.6^{\circ}\right)$. A deteriorated amplitude and phase profile can be observed in Fig. 9, where no amplitude singularity is formed. Based on the results shown in Figs. 8 and 9, it is evident that the performance of the OAM mode is significantly improved by using a smoother aperture phase distribution. Similar results in other operating modes are also observed although only one OAM mode is given in Fig. 9 for brevity.

\section{BANDWIDTH CHARACTERISTIC AND LINE-OF-SIGHT LINK EXPERIMENT}

Impedance bandwidth is usually adopted in constrained-fed OAM antennas to evaluate their bandwidth of functional operation [12]-[19] since their input impedances are critically affected by the frequency. However, no impedance mismatch occurs for DDL antennas within a wide frequency band due to their space feeding nature. As a result, a new definition of OAM 


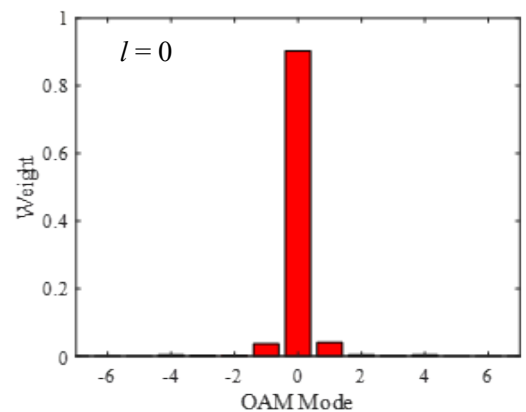

(a)

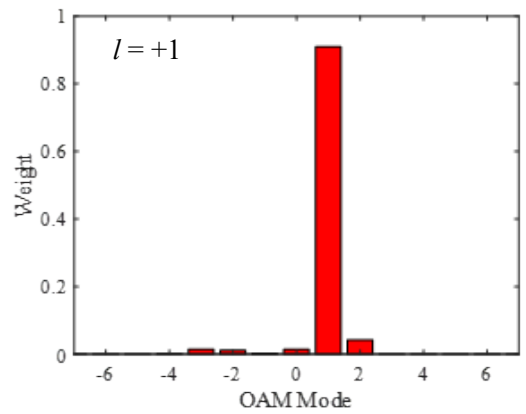

(b)

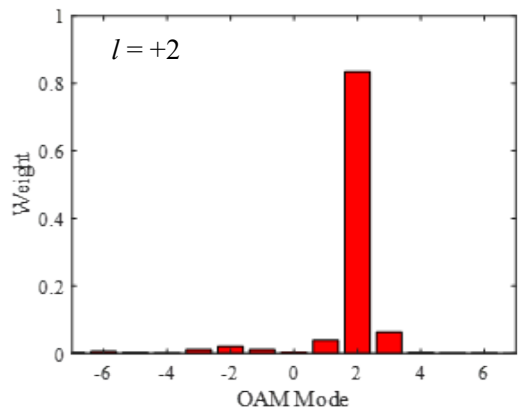

(c)

Fig. 11. Histograms of the simulated OAM spectrum weight for OAM mode number (a) $l=0$, (b) $l=+1$ and (c) $l=+2$ at $300 \mathrm{GHz}$, respectively.

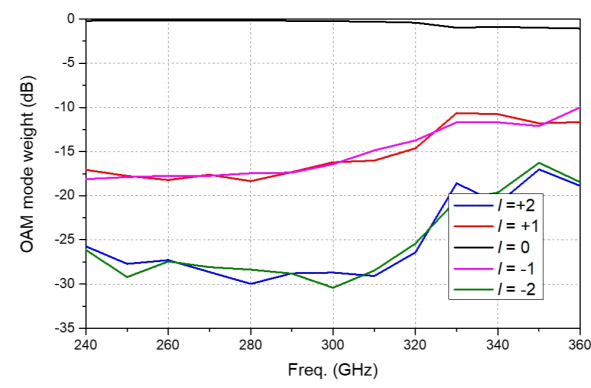

(a)

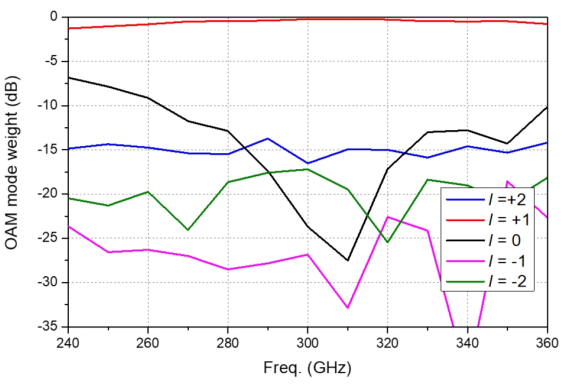

(b)

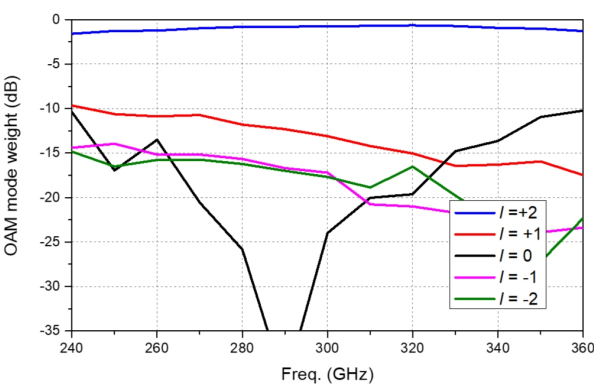

(c)

Fig. 12. Simulated weight of different OAM mode as a function of frequency for the DDL antennas which are reconfigured to realize OAM number (a) $l=0$, (b) $l$ $=+1$ and (c) $l=+2$, respectively. OAM mode weight $=20 \lg \left(\left|a_{l}\right|\right)$.
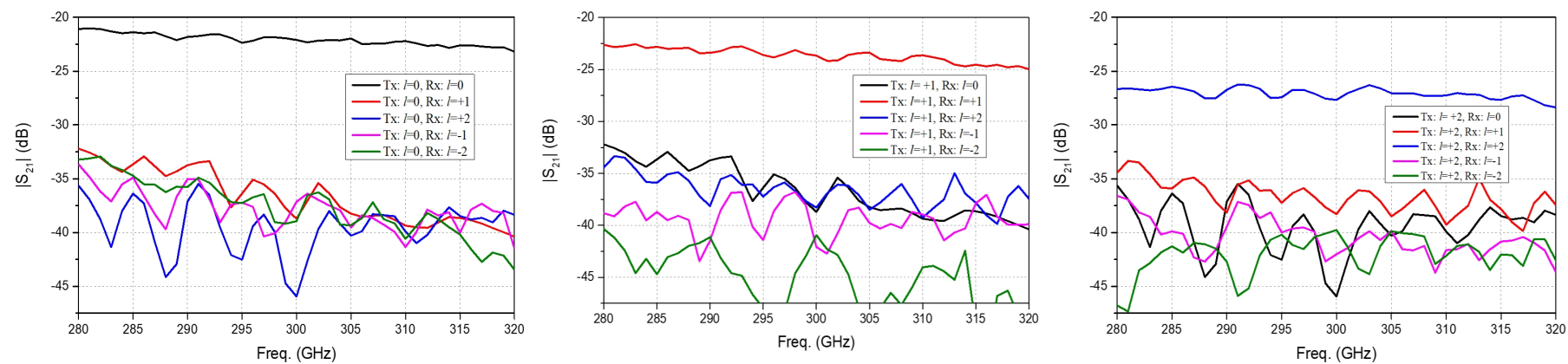

Fig. 13. Measured $\left|\mathrm{S}_{21}\right|$ of the line-of-sight link when the transmitting antenna is operating at OAM mode number (a) $l=0$, (b) $l=+1$ and (c) $l=+2$ and the receiving antenna is reconfigured to switch its operating OAM mode number among $l=0, \pm 1$, and \pm 2 .

bandwidth should be proposed to assess the bandwidth of the OAM antenna. OAM purity is an important figure of merit for vortex beams. Taking the OAM mode diversity applications as an example, high OAM purity is required to prevent energy coupling to channels with different $l$ charges and thereby reduce the inter-channel crosstalk. To analyze the OAM purity, the radiated field of the OAM generator can be decomposed into a superposition of a series of integer OAM modes, i.e., OAM spectrum, which can be obtained by Fourier transform of the field distribution $u(\varphi)$ on a circle centered on the beam axis [48]:

$$
a_{l}=\frac{1}{\sqrt{2 \pi}} \int_{0}^{2 \pi} u(\varphi) e^{-j l \varphi} d \varphi
$$

Fig. 11 shows the simulated OAM spectrum distributions for the cases of $l=0,+1$ and +2 at $300 \mathrm{GHz}$. It can be observed that the desired topological charge is the dominate mode with weights of $90.3 \%, 90.8 \%$ and $83.4 \%$ for the cases $l=0,+1$ and +2 , respectively. Fig. 12 shows the different simulated OAM mode weights as a function of frequency for the cases $l=0,+1$ and +2 . Here, we define the OAM mode purity bandwidth, i.e., the range of frequencies where the OAM mode isolations are within an acceptable value (10 dB is adopted in this work). From Fig. 12, the simulated OAM mode purity bandwidths of the designed DDL are larger than $40 \%$ (from 240 to $360 \mathrm{GHz}$ ), $31.3 \%$ (from 266 to $358 \mathrm{GHz}$ ) and $25.8 \%$ (from 272 to 349 $\mathrm{GHz}$ ) for the cases $l=0,+1$ and +2 , respectively. It can be seen that the DDL antenna can realize wideband OAM beam, which is attributed to the employed non-resonant dielectric post element as introduced in Section III.

To demonstrate the designed DDL antenna for OAM mode diversity applications, a $\mathrm{THz}$ near-field line-of-sight link is built as shown in Fig. 10. Two of the proposed DDL antennas are connected to the left and right OML extenders to work as the transmitter and receiver, respectively. The distance between the two DDL antennas is set as $50 \mathrm{~mm}\left(50 \lambda_{0}\right.$ at $\left.300 \mathrm{GHz}\right)$ and they are carefully aligned by using laser. The operating OAM modes of the two DDL antennas are switched by manually rotating their upper lens. The transmission coefficient of the 
two antennas with different OAM modes can be measured by the VNA and the measured results from 280 to $320 \mathrm{GHz}$ are given in Fig. 13. The measured frequency range is limited by the available $\mathrm{THz}$ measurement facilities. It can be clearly observed that much higher amplitudes of the transmission coefficient can be achieved when the two antennas are operated in the same OAM mode. Specifically, the mode isolations are better than $15 \mathrm{~dB}, 13 \mathrm{~dB}$ and $10 \mathrm{~dB}$ when the OAM modes of the DDL antennas are in $l=0, \pm 1$ and \pm 2 , respectively. The decrease of the OAM mode isolation for higher OAM mode number case is caused by the larger aperture phase errors in the azimuth range $\varphi \in\left[2 \pi-\varphi_{U}, 2 \pi\right]$ with a larger rotation of the upper DDL. Although a lower channel isolation for the case $l=$ \pm 2 is observed due to the two phase singularities shown in Fig. $\underline{8}$, it is still acceptable for the application of OAM-based wireless communications.

\section{CONCLUSION}

An OAM mode-reconfigurable DDL antenna operating at $\mathrm{THz}$ frequencies is presented in this paper. The operating OAM mode number among $l=0, \pm 1$, and \pm 2 can be switched by a simple mechanical in-plane rotation of the upper dielectric lens with specific angles relative to the lower one. During DDL element design, special attentions are paid to the fabrication sensitivity of the element structure for successfully fabricating the antenna in $\mathrm{THz}$ band. To alleviate the shadow effects of the employed height-variable dielectric post element, a smooth aperture phase distribution is developed by exploring the even-function nature of the phase-azimuth relationship of the required aperture compensation phase distribution. The $\mathrm{THz}$ DDL is conveniently fabricated by the in-house developed 3-D printing technology. Simulation and measurement results have verified the OAM mode-reconfigurability of the proposed $\mathrm{THz}$ DDL antenna.

\section{REFERENCES}

[1] L. Allen, M. W. Beijersbergen, R. Spreeuw, and J. Woerdman, "Orbital angular momentum of light and the transformation of Laguerre-Gaussian laser modes," Phys. Rev. A, Gen. Phys., vol. 45, no. 11, pp. 8185-8189, Jun. 1992.

[2] O. Edfors and A. J. Johansson, "Is orbital angular momentum (OAM) based radio communication an unexploited area?," IEEE Trans. Antennas Propag., vol. 60, no. 2, pp. 1126-1131, Feb. 2012.

[3] G. A. Turnbull, D. A. Robertson, G. M. Smith, L. Allen, M. J. Padgett,"The generation of free-space Laguerre-Gaussian modes at millimetre-wave frequencies by use of a spiral phas-eplate," Opt Commun., vol. 127, pp. 183-188, 1996.

[4] M. W. Beijersbergen, R. Coerwinkel, M. Kristensen, and J. P. Woerdman, "Helical-wavefront laser beams produced with a spiral phaseplate," Opt. Commun., vol. 112, pp. 321-327, 1994.

[5] M. Massari, G. Ruffato, M. Gintoli, F. Ricci, and F. Romanato, "Fabrication and characterization of high-quality spiral phase plates for optical applications," Appl. Opt., vol. 54, pp. 4077-4083, 2015.

[6] L. Cheng, W. Hong, and Z. Hao, "Generation of electromagneticwaves with arbitrary orbital angular momentum modes," Sci. Rep.,vol.4,p. 4814, 2014.

[7] D. Zelenchuk and V. Fusco, "Split-ring FSS spiral phase plate," IEEE Antennas Wireless Propag. Lett., vol. 12, pp. 284-287, 2013.

[8] P. Genevet et al., "Ultra-thin plasmonic optical vortex plate based on phase discontinuities," Appl. Phys. Lett., vol. 100, p. 013101, 2012.
[9] W. J. Byun, et al., "Simple generation of orbital angular momentum modes with azimuthally deformed cassegrain subreflector," Electron. Lett., vol. 51, no. 19, pp. 1480-1482, 2015.

[10] E. Mari, et al., "Near-field experimental verification of separation of OAM channels," IEEE Antennas Wireless Propag. Lett., vol. 14, pp. 556-558, 2015.

[11] X. Y. Lei and Y. J. Cheng, "High-efficiency and high-polarization separation reflectarray element for OAM-folded antenna application," IEEE Antennas Wireless Propag. Lett, vol. 16, pp. 1357-1360, 2017.

[12] B. Thidé, et al., "Utilization of photon orbital angular momentum in the low-frequency radio domain," Phys. Rev. Lett., vol. 99, no. 8, p. 087701, Aug. 2007.

[13] Q. Bai, A. Tennant and B. Allen, "Experimental circular phased array or generating OAM radio beams," Electron. Lett., vol. 50, no. 20, pp. 1414-1415, 2014.

[14] H. Li, L. Kang, F. Wei, Y.-M. Cai, and Y.-Z. Yin, "A low-profile dual-polarized microstrip antenna array for dual-mode OAM applications," IEEE Antennas Wireless Propag. Lett., vol. 16, pp. 3022-3025, 2017

[15] Y. Pan, et al., "Generation of orbital angular momentum radio waves based on dielectric resonator antenna," IEEE Antennas Wireless Propag. Lett., vol. 16, pp. 385-388, 2017.

[16] J. Ren and K. W. Leung, "Generation of microwave orbital angular momentum states using hemispherical dielectric resonator antenna," Appl. Phys. Lett., vol. 112, 2018, Art. no. 131103.

[17] A. Tennant and B. Allen, "Generation of OAM radio waves using circular time-switched array antenna," Electron. Lett., vol. 48, no. 21, pp. 1365-1366, 2012.

[18] C. Sun, S. Yang, Y. Chen, J. Guo, and S. Qu, "Realization of multiple orbital angular momentum modes simultaneously through four-dimensional antenna arrays," Sci. Rep., vol. 8, no. 1, pp. 149, 2018.

[19] S. Zheng, X. Hui, X. Jin, H. Chi and X. Zhang, "Transmission characteristics of a twisted radio wave based on circular traveling-wave antenna," IEEE Trans. Antennas Propag., vol. 63, no. 4, pp. 1530-1536, Apr. 2015.

[20] B. Allen, A. Tennant, Q. Bai, and E. Chatziantoniou, "Wireless data encoding and decoding using OAM modes," Electron. Lett., vol. 50, no. 3, pp. 232-233, Jan. 2014.

[21] K. Liu, et al., "Generation of orbital angular momentum beams for electromagnetic vortex imaging," IEEE Antennas Wireless Propag. Lett., vol. 15, pp. 1873-1876, 2016.

[22] K. Liu, et al., "Super-resolution radar imaging based on experimental OAM beams," Appl. Phys. Lett., vol. 110, no. 16, Apr. 2017, Art. no. 164102.

[23] M. P. Lavery, et al., "Detection of a spinning object using light's orbital angular momentum," Science, vol. 341, no. 6145, pp. 537-540, Aug. 2013.

[24] Y. Liu, G. Lin, Y. Cui, and R. Li, “An orbital angular momentum (OAM) mode reconfigurable antenna for channel capacity improvement and digital data encoding," Sci. Rep., vol. 7, p. 9852, Aug. 2017.

[25] Q. Liu, Z. N. Chen, Y. Liu, F. Li, Y. Chen, and Z. Mo, "Circular polarization and mode reconfigurable wideband orbital angular momentum patch array antenna," IEEE Trans. Antennas Propag., vol. 66, no. 4, pp. 1796-1804, Apr. 2018.

[26] L. Kang, H. Li, J. Zhou, S. Zheng and S. Gao, "A mode-reconfigurable orbital angular momentum antenna with simplified feeding scheme," IEEE Trans. Antennas Propag., vol. 67, no. 7, pp. 4866-4871, Jul. 2019.

[27] J. Wu, Z. Zhang, X. Ren, Z. Huang and X. Wu, "A broadband electronically mode-reconfigurable orbital angular momentum metasurface antenna," IEEE Antennas Wireless Propag. Lett., vol. 18, no. 7, pp. 1482-1486, Jul. 2019.

[28] L. Li and X. Zhu, "Mechanically reconfigurable single-arm spiral antenna array for generation of broadband circularly polarized orbital angular momentum vortex waves," Sci. Rep., vol. 8, p. 5128, Mar. 2018.

[29] Z. Chang, B. You, L. Wu, M. Tang, Y. Zhang and J. Mao, "A reconfigurable graphene reflectarray for generation of vortex $\mathrm{THz}$ waves," IEEE Antennas Wireless Propag. Lett., vol. 15, pp. 1537-1540, 2016. 
[30] Y. Shi and Y. Zhang, "Generation of wideband tunable orbital angular momentum vortex waves using graphene metamaterial reflectarray," IEEE Access, vol. 6, pp. 5341-5347, 2018.

[31] H. Yi, S. W. Qu, K. B. Ng, C. H. Chan, and X. Bai, "3-D printed millimeter-wave and terahertz lenses with fixed and frequency scanned beam," IEEE Trans. Antennas Propag., vol. 64, no. 2, pp. 442-449, Feb. 2016.

[32] G. Wu, Y. Zeng, K. F. Chan, S. Qu and C. H. Chan, "3-D printed terahertz lens with circularly polarized focused near field," 13th European Conference on Antennas and Propagation (EuCAP), Krakow, Poland, 2019, pp. 1-4.

[33] G. B. Wu, Y. Zeng, K. F. Chan, S. Qu and C. H. Chan, "High-gain circularly plarized lens antenna for terahertz applications," IEEE Antennas Wireless Propag. Lett., vol. 18, no. 5, pp. 921-925, May 2019.

[34] G. B. Wu, Y. S. Zeng, K. F. Chan, S. W. Qu and C. H. Chan, "3-D printed circularly polarized modified Fresnel lens operating at terahertz frequencies," IEEE Trans. Antennas Propag., vol. 67, no. 7, pp. 4429-4437, Jul. 2019.

[35] G. B. Wu, S. W. Qu, S. W. Yang, and C. H. Chan, "Broadband, singlelayer dual circularly polarized reflectarrays with linearly polarized feed," IEEE Trans. Antennas Propag., vol. 64, no. 10, pp. 4235-4241, Oct. 2016.

[36] G. B. Wu, S. W. Qu and S. Yang, "Wide-angle beam-scanning reflectarray with mechanical steering," IEEE Trans. Antennas Propag., vol. 66, no. 1, pp. 172-181, Jan. 2018.

[37] G. B. Wu, S. W. Qu, Y. X. Wang and S. W. Yang, "Nonuniform FSS-backed reflectarray with synthesized phase and amplitude distribution," IEEE Trans. Antennas Propag., vol. 66, no. 12, pp. 6883-6892, Dec. 2018.

[38] N. Gagnon and A. Petosa, "Using rotatable planar phase shifting surfaces to steer a high-gain beam," IEEE Trans. Antennas Propag., vol. 61, no. 6, pp. 3086-3092, Jun. 2013.

[39] M. U. Afzal and K. P. Esselle, "Steering the beam of medium-to-high gain antennas using near-field phase transformation," IEEE Trans. Antennas Propag., vol. 65, no. 4, pp. 1680-1690, Apr. 2017.

[40] M. Abd-Elhady, W. Hong and Y. Zhang, "A Ka-band reflectarray implemented with a single-layer perforated dielectric substrate," IEEE Antennas Wireless Propag. Lett., vol. 11, pp. 600-603, 2012.

[41] A. Massaccesi, et al., "3D-printable dielectric transmitarray with enhanced bandwidth at millimeter-waves," IEEE Access, vol. 6, pp. 46407-46418, 2018.

[42] Formlabs. Accessed: Jan. 2020. [Online]. Available: https://formlabs.com

[43] K. B. Ng and C. H. Chan, "On the dielectric properties of substrates with different surface conditions for submillimeter-wave and terahertz applications," THz Sci. Technol., vol. 9, no. 2, pp. 45-59, June 2016.

[44] X. X. Ruan and C. H. Chan, "Terahertz freespace dielectric property measurements using time- and frequency-domain setups," Int $J R F$ Microw. Comput. Aided Eng. e21839, 2019.

[45] S. Zhang, "Design and fabrication of 3D-printed planar Fresnel zone plate lens," Electron. Lett., vol. 52, no. 10, pp. 833-835, 1252016.

[46] S. Zhang, "Three-dimensional printed millimetre wave dielectric resonator reflectarray," IET Microw., Antennas \& Propag., vol. 11, no. 14, pp. 2005-2009, 19112017.

[47] A. Petosa and A. Ittipiboon, "Shadow blockage effects on the aperture efficiency of dielectric Fresnel lens," Inst. Elect. Eng. Proc., Microw., Antennas Propag., vol. 147, pp. 451-454, Dec. 2000.

[48] G. Molinaterriza, J. P. Torres, L. Torner, "Management of the angular momentum of light: preparation of photons in multidimensional vector states of angular momentum," Phys. Rev. Lett., vol. 88, no. 1, p. 013601, 2002. 